\section{A timely guide to the genome}

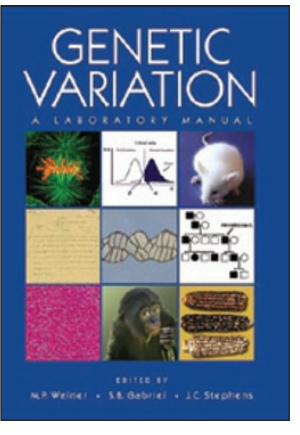

\section{Genetic Variation: A Laboratory Manual}

Edited by Michael P. Weiner, Stacey B. Gabriel \& J. Claiborne Stephens

Cold Spring Harbor Laboratory Press, 2007

472 pp., paperback, $\$ 165$

ISBN 978-087969780-8

\section{Reviewed by Eric E Schadt}

Writing a laboratory manual on genetic variation in the golden era of genetics, an era in which the rate of scientific discovery is beyond compare to that in any other point in history, is perhaps a bit like trying to give investment advice in today's quickly changing world markets. Considered in this light, the laboratory manual Genetic Variation is an impressive accomplishment. Not only did this editorial team assemble an impressive group of accomplished scientists as authors, but they produced a book of broad scope, covering a wide range of topics. In fact, it is the amazing breadth of this book that will ensure its relevance for some time to come. Although the technologies to generate data on large scales may change rapidly, other issues covered in this book related to consent, experimental design and the analysis and interpretation of high-dimensional data will change far more slowly, if at all.

The book is broken up into five sections covering study design; laboratory protocols for preparing RNA and DNA and then assessing SNP, copy number and more complex variations in these samples; data analysis; genetic variation studies carried out in model organisms; and insights into human DNA variation. In a refreshing departure from more traditional laboratory manuals, the book begins by addressing the many ethical issues facing human genetic research. In an age where genotype, molecular and clinical phenotype data are being generated on massive scales and then dropped into public domain databases, it is important to remind novices and experts alike of their obligations regarding consent, privacy, and data-use issues for studies involving human subjects. Even beyond the use of genetic variation information in a clinical or forensic science setting, we are seeing direct-to-consumer services emerge (23andMe, for example) with a significant recreational component ("Where am I descended from?") where samples are also incredibly broadly consented for science research. If we want continued public support to collect these types of data, we must act responsibly. This type of chapter does not appear frequently enough in this type of reference.

Second only to the consideration of ethical issues are experimental design issues, a subject covered in the first section. Because massive

Eric E. Schadt is in the Department of Genetics, Rosetta Inpharmatics,

LLC, Seattle, Washington 98109, USA.

e-mail:eric_schadt@merck.com amounts of genomic data can now be generated cheaply and efficiently via outsourcing to core laboratories, almost any basic science group can carry out studies of genetic variation. But generating data quickly and cheaply will not make up for poorly designed studies. Forming the appropriate hypotheses to be tested, identifying the best populations available to address such hypotheses and performing power calculations to ensure a study is appropriately powered are some of the topics discussed in this section, reminding the expert and providing the novice with invaluable insights into the important design components of any study.

The second section of this book does an excellent job providing a comprehensive guide on generating data in a variety of genetic variation experiments. Many state-of-the-art techniques are covered in three parts in this section, with a first set of chapters detailing DNA and RNA isolation techniques. A second set of chapters details intermediate-tolarge scale genotyping assays available for SNP-based markers, and a third set of chapters focuses on the detection of copy number variations using comparative genomic hybridization methods and complex variation analysis.

The third section will be most useful to the less experienced researcher, given its focus on data analysis issues facing those attempting to interpret hundreds of thousands or even millions of markers genotyped in thousands of individuals and their association to biological trait data. From the selection of SNPs covered in the first two chapters to the assessment of significance covered by the fourth chapter in this section, failing to address these important issues before designing a genetics study could lead to unnecessary limitations in addressing hypotheses of interest, or worse, to a misinterpretation of the results that would then motivate time-consuming follow-up studies based on erroneous conclusions. The chapter by Daly provides the beginning steps that must be understood to successfully analyze data in any genetic association study.

The fourth section provides a broad sampling of applications in model organisms that have relatively complete genomic information available. Covering several major plant (Arabidopsis, maize and rice) and animal (mouse, rat, cat, dog and chimpanzee) species, a wealth of information is provided relating to the history of the species in scientific research, the state of the genome for a given species, phenotypes studied and resources available for scientific research that in many cases are specific to a given species. The final section in this book focuses on insights into human variations, including a chapter on genealogical markers that are now finding use not only in the study of the origins of the Polynesians and other cultures (chapter 33 by Stoneking and Kayser), but also in studies with a strong recreational genetics component. In addition, there is an interesting chapter by Butler on the application of genetics in forensic science, highlighting many issues not discussed in the popular TV series CSI.

This manual is relevant on many different levels and will no doubt provide any interested reader with a wealth of useful information. I for one have added this book to my collection of references on carrying out genetic studies on the underlying causes of disease and drug response in human and mouse populations.

\section{COMPETING INTERESTS STATEMENT}

The author declares competing financial interests: details accompany the full-text HTML version of the paper at http://www.nature.com/naturegenetics/. 